\title{
Overcoming multidrug resistance by activating unfolded protein response of the endoplasmic reticulum in cisplatin-resistant A2780/CisR ovarian cancer cells
}

\author{
Euitaek Jung ${ }^{1}$, Dongsoo $\mathrm{Koh}^{2}$, Yoongho Lim ${ }^{3}$, Soon Young Shin ${ }^{1,4}$ E Young Han Lee ${ }^{1,4, *}$ \\ ${ }^{1}$ Department of Biological Sciences, Sanghuh College of Life Sciences, Konkuk University, Seoul 05029, ${ }^{2}$ Department of Applied Chemistry, \\ Dongduk Women's University, Seoul 02748, ${ }^{3}$ Division of Bioscience and Biotechnology, BMIC, Konkuk University, Seoul 05029, ${ }^{4}$ Cancer \\ and Metabolism Institute, Konkuk University, Seoul 05029, Korea
}

Cisplatin is a widely used anti-cancer agent. However, the effectiveness of cisplatin has been limited by the commonly developed drug resistance. This study aimed to investigate the potential effects of endoplasmic reticulum (ER) stress to overcome drug resistance using the cisplatin-resistant A2780/CisR ovarian cancer cell model. The synthetic chalcone derivative (E)-3-(3,5-dimethoxyphenyl)-1-(2-methoxyphenyl)prop2-en-1-one (named DPP23) is an ER stress inducer. We found that DPP23 triggered apoptosis in both parental cisplatinsensitive A2780 and cisplatin-resistant A2780/CisR ovarian cancer cells due to activation of reactive oxygen species (ROS)-mediated unfolded protein response (UPR) pathway in the endoplasmic reticulum. This result suggests that ROSmediated UPR activation is potential in overcoming drug resistance. DPP23 can be used as a target pharmacophore for the development of novel chemotherapeutic agents capable of overcoming drug resistance in cancer cells, particularly ovarian cancer cells. [BMB Reports 2020; 53(2): 88-93]

\section{INTRODUCTION}

Ovarian cancer is one of the most leading causes of death from gynecologic cancer worldwide. Cisplatin (Fig. 1A), cis-diamminedichloridoplatinum (II), is a platinum-based chemotherapeutic agent, which is commonly used in the treatment of many solid cancers, in particular, ovarian cancer (1). The primary mode of action of cisplatin is the formation of DNA adducts that induce DNA damage-mediated apoptosis.

*Corresponding author. Tel: +82-2-2049-6115; Fax: +82-3437-9781; E-mail: yhlee58@konkuk.ac.kr

https://doi.org/10.5483/BMBRep.2020.53.2.108

Received 15 April 2019, Revised 10 May 2019, Accepted 3 June 2019

Keywords: Cisplatin resistance, DPP23, Ovarian cancer, Reactive oxygen species, Unfolded protein response
The efficacy of cisplatin and other platinum-based derivatives is high at early stages, but the majority of patients develop drug resistance at advanced stages (2). However, despite intensive efforts to develop chemotherapeutic agents, the number of potential chemotherapeutic drugs overcoming drug resistance in ovarian cancer is limited at present. Therefore,

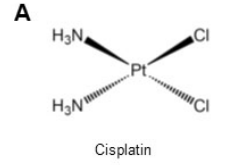

B

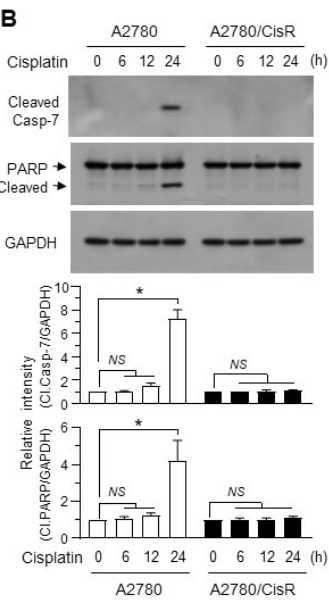

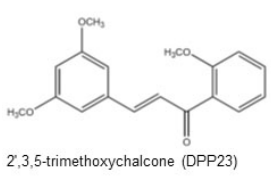
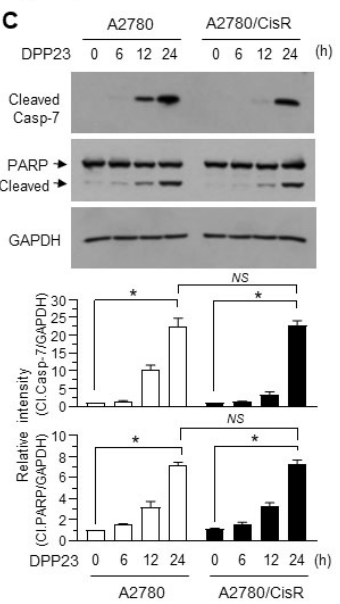

Fig. 1. Effect of DPP23 on the activation of caspase-7 in cisplatinresistant ovarian cancer cells. (A) Chemical structures of cisplatin and DPP23. (B, C) A2780 and A2780/CisR cells treated with (B) $1 \mu \mathrm{g} / \mathrm{ml}$ cisplatin or (C) $10 \mu \mathrm{M}$ DPP23 for different time periods. Immunoblot analysis was performed using specific antibodies against cleaved caspase-7 and poly (ADP-ribose) polymerase (PARP). Glyceraldehyde 3-phosphate dehydrogenase (GAPDH) was used as a loading control. The band intensities of cleaved caspase-7 and PARP were measured relative to GAPDH levels using ImageJ software. The data are presented as means \pm SD ( $n$ $=3)$. NS, not significant; $* P<0.001$; according to Sidak's multiple comparisons test. 
there is a need to design and develop additional chemotherapeutic agents that can overcome drug resistance in ovarian cancer.

The mechanisms of cisplatin resistance include overexpression of microRNA (3), loss of the tumor suppressor TP53 gene function and increased levels of cellular glutathione levels by cisplatin (2). A synthetic methoxy chalcone derivative, (E)-3-(3,5-dimethoxyphenyl)1-(2-methoxyphenyl)prop-2-en-1-one (2',3,5-trimethoxychalcone, named DPP-23; Fig. 1A) (3), contains a Michael-acceptor moiety which reacts with thiols in glutathione, leading to a decrease in intracellular glutathione levels (4). We previously demonstrated that DPP23 induces apoptosis independently of the p53 genetic status of the cancer cells, but not in non-transformed cells, via generation of reactive oxygen species (ROS) and the endoplasmic reticulum (ER) stress-activated unfolded protein response (UPR) pathway (3). The previous study has demonstrated that combined treatment with HIV protease inhibitor nelfinavir and the cyclooxygenase-2 (COX-2) inhibitor celecoxib enhances killing of drug-resistant breast cancer cells through the ER stress responses (5). The present study aimed to evaluate whether the activation of ROS-induced UPR by DPP23 could overcome drug resistance in cisplatin-resistant ovarian cancer cells. Here, we found that DPP23 triggers apoptosis in both cisplatin-sensitive parental A2780 and cisplatin-resistant A2780/CisR ovarian cancer cells.

\section{RESULTS AND DISCUSSION}

\section{DPP23 activates caspase-7 in A2780/CisR cells}

In order to test the drug-resistant property of A2780/CisR cells, we treated parental A2780 and cisplatin-resistant A2780/CisR ovarian cancer cells with cisplatin. Caspase-7 is an executioner caspase that is cleaved and activated by the initiator caspases, such as caspase-9, during apoptosis (6). Immunoblot analysis revealed that exposure to cisplatin significantly $(\mathrm{P}<0.001$ by Sidak's multiple comparisons test, $n=3$ ) increased the cleavage of caspase-7 and its substrate protein, poly (ADP-ribose) polymerase (PARP) in parental cisplatin-sensitive A2780 ovarian cancer cells, but not in cisplatin-resistant A2780/CisR cells (Fig. $1 \mathrm{~B})$, confirming that $\mathrm{A} 2780 / \mathrm{CisR}$ cells show resistance to cisplatin.

We tested whether the ER stress inducer DPP23 can overcome cisplatin resistance. Fig. 1C shows that DPP23 enhanced the accumulation of cleaved forms of both caspase7 and PARP in A2780/CisR cells, which was statistically significant after $24 \mathrm{~h}$ ( $\mathrm{P}<0.0001$ by Sidak's multiple comparisons test, $n=3$ ). The differences between A2780 and A2780/CisR cells in caspase-7 and PARP cleavages were not statistically significant $(\mathrm{P}>0.05$ by Sidak's multiple comparisons test, $n$ $=3$ ) after $24 \mathrm{~h}$ of DPP23 treatment, suggesting that DPP23 overcame cisplatin resistance in A2780/CisR cells.

\section{DPP23 activates caspase-7 in MES-SA/DX5 cells}

Next, we asked whether DPP23 can stimulate the caspase pathway in a different drug-resistant cancer cell line, MES-SA/DX5, which are multidrug-resistant human uterine sarcoma cells overexpressing P-glycoprotein (7). We confirmed that treatment with cisplatin stimulated the cleavages of caspase-7 and PARP in drug-sensitive MES-SA cells but observed negligible changes in drug-resistant MES-SA/DX5 cells (Fig. 2A). However, DPP23 significantly ( $\mathrm{P} \leq 0.001$ by Sidak's multiple comparisons test, $n=3$ ) stimulated the cleavages of caspase-7 and PARP in both MES-SA and MES-SA/DX5 cells (Fig. 2B). No significant change was observed between MES-SA and MES-SA/DX5 cells after a 24-h treatment with DPP23. These data suggested that UPR inducer DPP23 could be broadly effective in overcoming drug resistance of cancer cells.

\section{DPP23 triggers apoptosis through generation of reactive oxygen species in A2780/CisR cells}

In general, polyphenols appear to have antioxidant properties (8). However, some natural flavonoids, including quercetin and resveratrol, produce ROS, which triggers oxidative stress and apoptosis in cancer cells $(9,10)$. It has been reported that the redox active Schiff's base, potassium-N-(2-hydroxy-3-methoxybenzaldehyde)-alaninate, is associated with ROS-mediated anti-tumor activity in doxorubicin-resistant T-lymphoblastic leukemia cells (11). We have previously demonstrated that
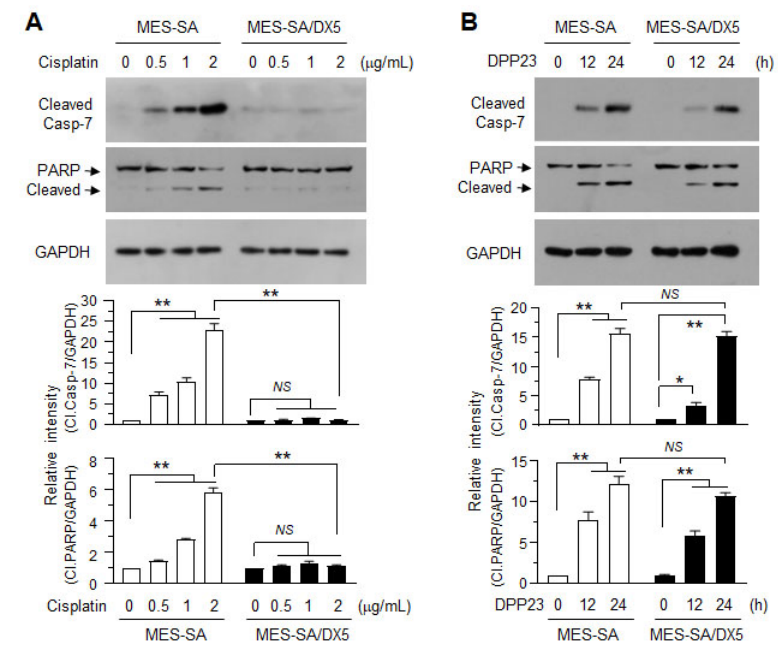

Fig. 2. Effect of DPP23 on the activation of caspase-7 in multidrugresistant uterine sarcoma cells. MES-SA and MES-SA/DX5 cells were treated with (A) cisplatin at different concentrations or (B) $10 \mu \mathrm{M}$ DPP23 for various time periods. Immunoblot analysis was performed using specific antibodies against cleaved caspase-7 and poly (ADP-ribose) polymerase (PARP). Glyceraldehyde 3-phosphate dehydrogenase (GAPDH) was used as a loading control. The band intensities of cleaved caspase-7 and PARP were measured relative to GAPDH levels using Image software. The data are presented as means \pm SD $(n=3)$. NS, not significant; $* * P<$ 0.001; according to Sidak's multiple comparisons test. 
DPP23 (3) and 2'-hydroxy-2,3,5'-trimethoxychalcone (12) induce apoptosis through generation of ROS. Based on these findings, we hypothesized that DPP23 efficacy in overcoming drug resistance of cancer cells could be associated with intracellular ROS generation. To test this hypothesis, we examined whether DPP23 could generate ROS in ovarian cancer cells by using a ROS-sensitive dye, 2', $7^{\prime}$-dichlorofluorescein diacetate (DCF-DA), as a fluorescent probe. Intracellular ROS was detected using fluorescence microscopy. DPP23 increased ROS generation in both $\mathrm{A} 2780$ and $\mathrm{A} 2780 /$ CisR cells and more efficiently in A2780/CisR cells than A2780 cells (Fig. 3A). Flow cytometry showed that DPP23-induced ROS production was maintained up to $10 \mathrm{~h}$ after exposure in both the cell lines (Fig. 3B).

We next investigated whether DPP23-induced ROS triggers apoptosis. $\mathrm{N}$-acetyl cysteine (NAC) confers antioxidant effects by reducing free radicals. Pretreatment of A2780/CisR cells

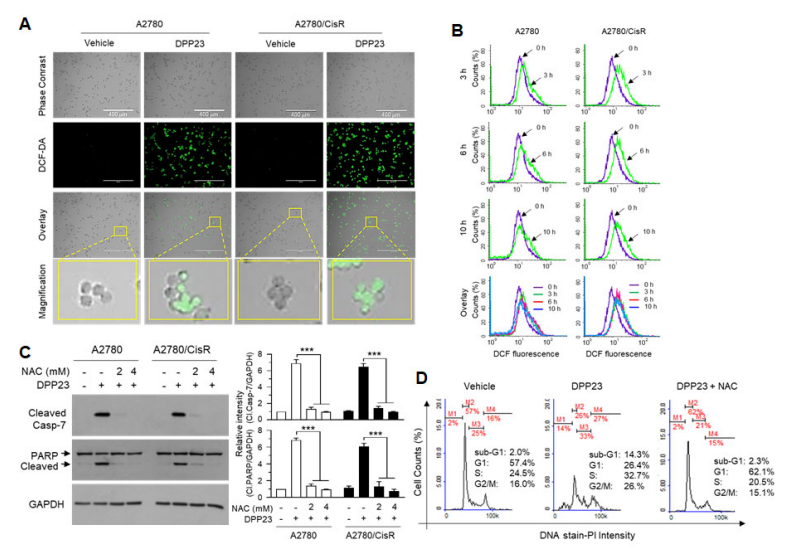

Fig. 3. Effect of DPP23 on reactive oxygen species (ROS)-mediated apoptosis. (A) A2780 and A2780/CisR cells were incubated with $10 \mu \mathrm{M}$ DCF-DA for $60 \mathrm{~min}$, followed by $10 \mu \mathrm{M}$ DPP23 for $3 \mathrm{~h}$. DCF fluorescence was detected using an EVOSf1 ${ }^{\circledR}$ fluorescence microscope. Scale bar, $400 \mu \mathrm{m}$. (B) DCF fluorescence was measured using a FACSCalibur flow cytometer. A2780 and A2780/CisR cells were treated with $10 \mu \mathrm{M}$ DCF-DA for $60 \mathrm{~min}$, followed by $10 \mu \mathrm{M}$ DPP23 for $0,3,6$, and $10 \mathrm{~h}$. DCF fluorescence was measured using a FACSCalibur flow cytometer. (C) A2780 and A2780/CisR cells were treated with $10 \mu \mathrm{M}$ DPP23 in the absence or presence of 2- or 4-mM N-acetyl cysteine (NAC). Whole cell lysates were prepared and immunoblot analysis was performed using specific antibodies against cleaved caspase-7 and poly (ADP-ribose) polymerase (PARP). Glyceraldehyde 3-phosphate dehydrogenase (GAPDH) was used as a loading control. The band intensities of cleaved caspase-7 and PARP were measured relative to GAPDH levels using Imagej software. The data are presented as means $\pm \mathrm{SD}(n=3)$. ${ }^{* * *} \mathrm{P}<0.001$; according to Sidak's multiple comparisons test. (D) A2780/CisR cells were treated with vehicle (DMSO) or $10 \mu \mathrm{M}$ DPP23 in the absence or presence of $4 \mathrm{mM} \mathrm{N}$-acetyl cysteine (NAC). Cells were fixed with ethanol and stained with propidium iodide (PI). PI fluorescence was measured using a NucleoCounter NC-3000. M1, sub-G1 phase; M2, G1 phase; M3, S phase; M4, G2/M phase. with NAC significantly ( $\mathrm{P}<0.001$ by Sidak's multiple comparisons test) abrogated DPP23-induced both caspase-7 and PARP cleavages (Fig. 3C). Flow cytometry was performed to confirm the induction of apoptosis. As shown in Fig. 3D, DPP23 increased the number of sub-G0/G1 phase cells (vehicle, $2.0 \%$ vs. DPP23, 14.3\%), a common indicator of cell death, accompanied with a decrease in G1 phase population (vehicle, $57.4 \%$ vs. DPP23, 26.4\%). NAC pretreatment ameliorated DPP23-induced accumulation of sub-G1 cells (DPP23, 14.3\% vs. DPP23 + NAC, $2.3 \%$ ). These data suggested that DPP23 triggers caspase-mediated cell death through the generation of ROS in both A2780 and A2780/CisR cells.

\section{DPP23 stimulates unfolded protein response in A2780/CisR cells}

DPP23 triggers endoplasmic reticulum (ER)-stress response in various cancer cells through ROS-mediated unfolded protein response (UPR) (3). We, therefore, investigated whether DPP23 stimulates the UPR pathway in A2780/CisR cells. Upon DPP23 treatment, the levels of ER-resident UPR sensor proteins, including the $78-k D a$ glucose-regulated protein (GRP78) and inositol-requiring enzyme 1-alpha (IRE1 $\alpha$ ), significantly increased after $12 \mathrm{~h}$ in both A2780 and A2780/CisR cells (all $P<0.05$ by Sidak's multiple comparisons test) (Fig. 4A). The X-box binding protein 1 (XBP1) is an ER stress-inducible transcription factor that regulates the expression of ER stress response genes. Its mRNA expression is regulated by unconventional mRNA splicing in the cytoplasm (13). Unspliced XBP1 (uXBP1) mRNA is expressed with an unspliced 26 nucleotide-sized intron which contains a restriction site for Pstl. Upon ER stress, activated IRE1 $\alpha$ removes the unspliced intron from the uXBP1 transcript to generate an active spliced form of XBP1 mRNA (sXBP1) (14). Thus, the presence of sXBP1 mRNA serves as a marker for ER stress. The cDNA from the polymerase chain reaction (PCR) amplicon of UXBP1 mRNA contains a Pstl restriction enzyme-cleavage sequence. The uXBP1 mRNA amplicon was cleaved by Pstl, resulting in the generation of 279 and 261 base-pair (bp) sequences (Fig. 4B, top). However, the sXBP1 amplicon that lacks the Pstl cleavage sequence is not digested by the Pstl, thereby producing a 540 bp sequence (Fig. 4B, bottom). We found that $\mathrm{UXBP} 1 \mathrm{mRNA}$ levels increased within $6 \mathrm{~h}$ after DPP23 treatment and then decreased by $24 \mathrm{~h}$ to below the basal level, whereas sXBP1 mRNA slightly increased at $6 \mathrm{~h}$, reached a maximum at $12 \mathrm{~h}$, and then decreased in both the cell lines (Fig. 4C). We also confirmed that protein levels of sXBP1 increased by DPP23 treatment in both the cell lines (Fig. 4D). These data suggested that DPP23 upregulates XBP1 mRNA expression and stimulates XBP1 mRNA splicing probably through IRE $1 \alpha$ upregulation. Collectively, it seems likely that DPP23 triggers ER stress response in both A2780 and A2780/CisR cells. Notably, sXBP1 accumulated more abundantly in A2780/CisR cells than in A2780 cells. Although further research is needed to 
A

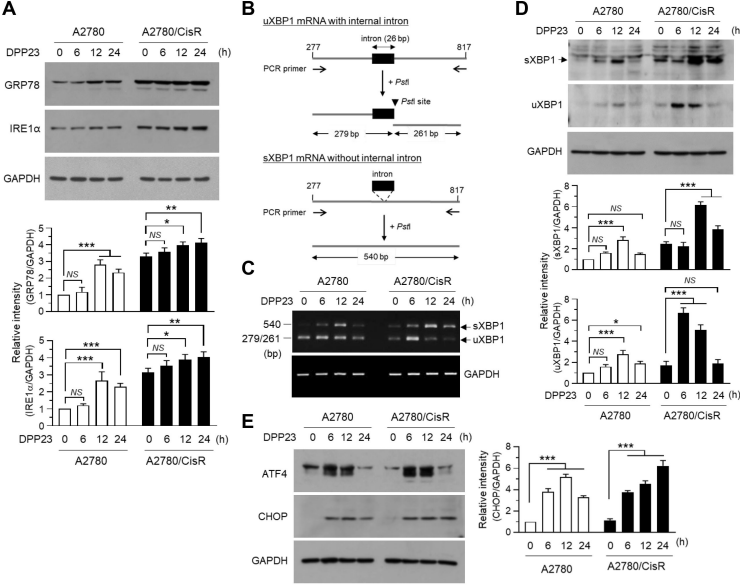

Fig. 4. Effect of DPP23 on the activation of the unfolded protein response (UPR) pathway. (A) A2780 and A2780/CisR cells were treated with $10 \mu \mathrm{M}$ DPP23 for various time periods. Whole cell lysates were prepared and immunoblot analysis was performed using antibodies against ER stress sensor proteins, GRP78 and IRE1 $\alpha$. GAPDH was used as an internal control. The band intensities of GRP78 and IRE1 $\alpha$ were measured relative to GAPDH levels using ImageJ software. The data are presented as means \pm SD $(n=$ 3). NS, not significant; ${ }^{*} P<0.05 ;{ }^{* * P}<0.01 ;{ }^{* * * P}<0.001$; according to Sidak's multiple comparisons test. (B) Schematic representation of unspliced XBP-1 (UXBP-1) and spliced XBP-1 (sXBP-1) mRNA. The arrow-head indicates the Pstl restriction site. Numbers denote nucleotide (nt) positions from the transcription start site. The internal intron site from nt 531 to nt 556 is indicated as a black box. (C) A2780 and A2780/CisR cells were treated with $10 \mu \mathrm{M}$ DPP23 for various time periods. After total RNA isolation, XBP-1 mRNA was amplified from nt 277 to $n t$ 817 by RT-PCR. Sizes of uXBP-1 (279 bp and 261 bp) and sXBP-1 (540 bp) mRNAs amplicons are indicated. GAPDH mRNA was amplified as the internal control. (D) A2780 and A2780/CisR cells were treated with $10 \mu \mathrm{M}$ DPP23 for various time periods. Whole cell lysates were subjected to immunoblotting using antibodies against XBP-1. GAPDH was used as an internal control. The band intensities of GRP78 and IRE1 $\alpha$ were measured relative to GAPDH levels using Image software. The data are presented as means $\pm \mathrm{SD}(n=3)$. NS, not significant; ${ }^{* P}<0.05 ; * * * \mathrm{P}<$ 0.001; according to Sidak's multiple comparisons test. (E) A2780 and A2780/CisR cells were treated with $10 \mu \mathrm{M}$ DPP23 for various time periods. Whole cell lysates were prepared and immunoblot analysis was performed using an antibody against CHOP and ATF4. GAPDH was used as an internal control. The band intensities of CHOP were measured relative to GAPDH levels using Imagej software. The data are presented as means \pm SD $(n=3) .{ }^{* * * P}<0.001 ;$ according to Sidak's multiple comparisons test.

understand this phenomenon, it appears that drug-resistant A2780/CisR cells are more sensitive to DPP23 than parental A2780 cells.

During ER stress, the activating transcription factor 4 (ATF4) induces C/EBP-homologous protein (CHOP), also known as DNA damage-inducible transcript 3 (DDIT3) or growth arrest and DNA damage-inducible protein (GADD153), a stress- inducible transcription factor that mediates ER stress-induced apoptosis (15). To determine whether DPP23 upregulates ATF4 and CHOP expression, A2780 and A2780/CisR cells were treated with $20 \mu \mathrm{M}$ DPP23 for various periods, and ATF4 and $\mathrm{CHOP}$ protein levels were determined by immunoblot analysis. Increase in ATF4 and CHOP levels was detected within 6 h (Fig. 4E). CHOP levels remained significantly $(\mathrm{P}<$ 0.001 by Dunnett's multiple comparisons test) elevated up to $24 \mathrm{~h}$ after DPP23 treatment (Fig. 4E). It has been reported that $\mathrm{CHOP}$ directly regulates pro-apoptotic genes, such as B-cell leukemia/lymphoma-2-like protein 11 (BCL2L11), a member of the BCL-2 family which promotes apoptosis through inhibition of BCL-2 and activation of initiator caspase-9 $(16,17)$. To further address the effect of DPP23 on ER stress response, we utilized an ER stress inhibitor, sodium 4-phenylbutyric acid (4-PBA). We found that pretreatment with 4-PBA prevented DPP23-induced cleavages of both caspase-7 and PARP in a dose-dependent manner (Supplementary Fig. S1). Thus, it seems likely that DPP23 triggers UPR-mediated apoptosis through the activation of the ATF4-CHOP-BCL2L11-caspase pathway.

In summary, DPP23 induces caspase-mediated apoptosis through the generation of ROS and the stimulation of the UPR pathway in both cisplatin-sensitive A2780 and cisplatin-resistant A2780/CisR ovarian cancer cells. Therefore, ROS-induced UPR activation might be a promising strategy for overcoming drug resistance in various cancer cells. We propose that the ER stress inducer DPP23 is an effective pharmacophore for the development of novel chemotherapeutic agents that can overcome the drug resistance of cancer cells, particularly ovarian cancer cells.

\section{MATERIALS AND METHODS}

\section{Cell culture}

Cisplatin-sensitive A2780 and cisplatin-resistant A2780/CisR ovarian cancer cells were obtained from the American Type Culture Collection (ATCC, Rockville, MD, USA). The cells were grown in Dulbecco's modified Eagle's medium (DMEM) supplemented with $10 \%(\mathrm{v} / \mathrm{v})$ fetal bovine serum (CellGro/ Corning, Manassas, VA, USA) at $37^{\circ} \mathrm{C}$ in a humidified $5 \% \mathrm{CO}_{2}$ incubator, as described previously (18).

\section{Cell cycle analysis}

The cell cycle phase was determined by measuring DNA contents using propidium iodide (PI) staining as described previously (19). Briefly, A2780 and A2780/CisR cells were treated with $10 \mu \mathrm{M}$ DPP23 for $24 \mathrm{~h}$, fixed in $70 \%(\mathrm{v} / \mathrm{v})$ ethanol, washed twice in phosphate-buffered saline (PBS), and then stained with $50 \mu \mathrm{g} / \mathrm{ml} \mathrm{Pl}$ containing $0.1 \%(\mathrm{v} / \mathrm{v})$ Triton $\mathrm{X}-100,0.1 \mathrm{mM}$ ethylenediaminetetraacetic acid (EDTA), and $50 \mu \mathrm{g} / \mathrm{ml}$ RNase A. Cells with diploid DNA (2N), tetraploid DNA (4N), between diploid and tetraploid, and lower than diploid were considered to be $\mathrm{G} 1, \mathrm{G} 2 / \mathrm{M}, \mathrm{S}$, and sub-G1 phase 
cells, respectively. The percentage of cells in each cell cycle phase was calculated by flow cytometry using a NucleoCounter NC-3000 (ChemoMetec, Allerød, Denmark), as described previously (19).

\section{Apoptosis assay using Annexin V staining}

Apoptosis was analyzed by detecting phosphatidylserine (PS) on the outer leaflet of the plasma membrane using a FITC-conjugated annexin V kit (BD Pharmingen; San Diego, CA, USA), according to the manufacturer's instructions. Dead cells were counterstained using propidium iodide (PI). Annexin V- and Pl-stained cells were counted via flow cytometry using a NucleoCounter NC-3000, as described previously (20).

\section{Immunoblot analysis}

Immunoblot analysis was performed as described previously (21). Briefly, cell lysates (10-20 $\mu \mathrm{g})$ were prepared, separated via electrophoresis by SDS-PAGE, and transferred to nitrocellulose blots. Antibody-reactive protein bands were visualized using an enhanced chemiluminescence detection system (Amersham Pharmacia Biotech Inc., Piscataway, NJ, USA). Antibodies against cleaved caspase-7 (Asp198), poly (ADP-ribose) polymerase (PARP), 78-kDa glucose-regulated protein (GRP78), ATF4, inositol-requiring enzyme 1-alpha (IRE1 $\alpha$ ), and C/EBP homologous protein (CHOP) were obtained from Cell Signaling Technology (Beverly, MA, USA). Antibodies against glyceraldehyde 3-phosphate dehydrogenase $(\mathrm{GAPDH})$ and $\mathrm{X}$-box binding protein 1 (XBP-1) were purchased from Santa Cruz Biotechnology (Santa Cruz, CA, USA). Relative band intensity normalized to GAPDH was measured using ImageJ version 1.52a software (National Institute of Health, MD, USA).

\section{Analysis of intracellular reactive oxygen species (ROS)}

Cells were trypsinized, and then their reactive oxygen species (ROS) content was determined by incubation with $10 \mu \mathrm{M}$ 2',7'-dichlorofluorescein diacetate (DCF-DA; Sigma-Aldrich, Merck, Damstadt, Germany) for $30 \mathrm{~min}$. Intracellular ROS were visualized under an EVOSf $1{ }^{\circledR}$ fluorescence microscope (Advance Microscopy Group, Bothell, WA, USA). Fluorescence intensity was measured using a FACSCalibur flow cytometer (BD Biosciences, San Jose, CA, USA).

\section{Analysis of XBP-1 splicing by Pstl digestion}

Total RNA was extracted using a Trizol RNA extraction kit (Invitrogen, Carlsbad, CA). cDNA was synthesized using an iScript cDNA synthesis kit (Bio-Rad, Hercules, CA). XBP1 mRNA between nt 277 and 817 was amplified using XBP1-specific primers (forward, 5'-AGAGTAGCAGCTCAG ACTGC-3'; reverse, 5'-CATTAATGGCTTCCAGCTTG-3'), as described previously (22). Recognition site of Pstl is located within the 26-nt intron (nt 531-556) that is lost after IRE1 $\alpha$-mediated splicing (23). XBP1 PCR amplicons were digested for $2 \mathrm{~h}$ at $37^{\circ} \mathrm{C}$ to distinguish between unspliced uXBP1 and spliced sXBP1s mRNAs. PCR product of unspliced uXBP1 mRNA was expected to be cleaved into two fragments of $279 \mathrm{bp}$ and $261 \mathrm{bp}$ by Pstl digestion, while that of spliced sXBP1 mRNA is resistant to Pstl digestion, producing a $540 \mathrm{bp}$ band.

\section{ACKNOWLEDGEMENTS}

This study was supported by the Konkuk University Research Support Program.

\section{CONFLICTS OF INTEREST}

The authors have no conflicting interests.

\section{REFERENCES}

1. Moore WR, Anderson ME, Meister A, Murata $K$ and Kimura A (1989) Increased capacity for glutathione synthesis enhances resistance to radiation in Escherichia coli: a possible model for mammalian cell protection. Proc Natl Acad Sci U S A 86, 1461-1464

2. Godwin AK, Meister A, O'Dwyer PJ, Huang CS, Hamilton TC and Anderson ME (1992) High resistance to cisplatin in human ovarian cancer cell lines is associated with marked increase of glutathione synthesis. Proc Natl Acad Sci U S A 89, 3070-3074

3. Shin SY, Lee JM, Lee MS et al (2014) Targeting cancer cells via the reactive oxygen species-mediated unfolded protein response with a novel synthetic polyphenol conjugate. Clin Cancer Res 20, 4302-4313

4. Adams DJ, Dai M, Pellegrino G et al (2012) Synthesis, cellular evaluation, and mechanism of action of piperlongumine analogs. Proc Natl Acad Sci U S A 109, 15115-15120

5. Cho HY, Thomas S, Golden EB et al (2009) Enhanced killing of chemo-resistant breast cancer cells via controlled aggravation of ER stress. Cancer Lett 282, 87-97

6. Boatright KM and Salvesen GS (2003) Mechanisms of caspase activation. Curr Opin Cell Biol 15, 725-731

7. Harker WG and Sikic BI (1985) Multidrug (pleiotropic) resistance in doxorubicin-selected variants of the human sarcoma cell line MES-SA. Cancer Res 45, 4091-4096

8. Miura YH, Tomita I, Watanabe T, Hirayama T and Fukui S (1998) Active oxygens generation by flavonoids. Biol Pharm Bull 21, 93-96

9. Gaspar J, Rodrigues A, Laires A et al (1994) On the mechanisms of genotoxicity and metabolism of quercetin. Mutagenesis 9, 445-449

10. Luo H, Yang A, Schulte BA, Wargovich MJ and Wang GY (2013) Resveratrol induces premature senescence in lung cancer cells via ROS-mediated DNA damage. PLoS One 8, e60065

11. Basu S, Ganguly A, Chakraborty P et al (2012) Targeting the mitochondrial pathway to induce apoptosis/necrosis through ROS by a newly developed Schiff's base to 
overcome MDR in cancer. Biochimie 94, 166-183

12. Lee $D H$, Jung Jung $Y$, Koh $D, \operatorname{Lim} Y$, Lee $Y H$ and Shin $S Y$ (2016) A synthetic chalcone, 2'-hydroxy-2,3,5'-trimethoxychalcone triggers unfolded protein response-mediated apoptosis in breast cancer cells. Cancer Lett 372, 1-9

13. Uemura A, Oku M, Mori $\mathrm{K}$ and Yoshida $\mathrm{H}$ (2009) Unconventional splicing of XBP1 mRNA occurs in the cytoplasm during the mammalian unfolded protein response. J Cell Sci 122, 2877-2886

14. Yoshida $\mathrm{H}$, Matsui T, Yamamoto A, Okada T and Mori $\mathrm{K}$ (2001) XBP1 mRNA is induced by ATF6 and spliced by IRE1 in response to ER stress to produce a highly active transcription factor. Cell 107, 881-891

15. Szegezdi E, Logue SE, Gorman AM and Samali A (2006) Mediators of endoplasmic reticulum stress-induced apoptosis. EMBO Rep 7, 880-885

16. Puthalakath $H, O^{\prime}$ Reilly LA, Gunn $P$ et al (2007) ER stress triggers apoptosis by activating $\mathrm{BH} 3$-only protein Bim. Cell 129, 1337-1349

17. O'Connor L, Strasser A, O'Reilly LA et al (1998) Bim: a novel member of the Bcl-2 family that promotes apoptosis. EMBO J 17, 384-395

18. Cao L, Wan Q, Li F and Tang Ce (2018) MiR-363 inhibits cisplatin chemoresistance of epithelial ovarian cancer by regulating snail-induced epithelial-mesenchymal transition. BMB Rep 51, 456-461

19. Shin SY, Yoon H, Ahn S et al (2013) Structural Properties of Polyphenols Causing Cell Cycle Arrest at G1 Phase in HCT116 Human Colorectal Cancer Cell Lines. Int J Mol Sci 14, 16970-16985

20. Shin SY, Yoon H, Ahn S et al (2013) Chromenylchalcones showing cytotoxicity on human colon cancer cell lines and in silico docking with aurora kinases. Bioorg Med Chem 21, 4250-4258

21. Shin SY, Lee KS, Choi YK et al (2013) The antipsychotic agent chlorpromazine induces autophagic cell death by inhibiting the $A k t / m T O R$ pathway in human U-87MG glioma cells. Carcinogenesis 34, 2080-2089

22. Gil HN, Koh D, Lim Y, Lee YH and Shin SY (2018) The synthetic chalcone derivative 2-hydroxy-3',5,5'-trimethoxychalcone induces unfolded protein response-mediated apoptosis in A549 lung cancer cells. Bioorg Med Chem Lett 28, 2969-2975

23. Calfon $M$, Zeng $H$, Urano $F$ et al (2002) IRE1 couples endoplasmic reticulum load to secretory capacity by processing the XBP-1 mRNA. Nature 415, 92-96 